\title{
Absolute position calculation for a desktop mobile robot based on three optical mouse sensors
}

\author{
Haritz Zabaleta, David Valencia, Joel Perry, Jan Veneman, Thierry Keller \\ Quality of Life Unit Biorobotics Department \\ Tecnalia Research and Innovation \\ Paseo Mikeletegi, 1, 20011 Donostia \\ Email: haritz.zabaleta@tecnalia.com
}

\begin{abstract}
ArmAssist is a wireless robot for after stroke upper limb rehabilitation. In this paper, we describe a method based on artificial landmark navigation system. The navigation system is only based in three optical mouse sensors. This enables to build a cheap but reliable position sensor. Two of the sensors are the data source for odometry calculations, and the third optical mouse sensor takes very low resolution pictures of a custom designed mat. These pictures are processed by an optical symbol recognition algorithm which will estimate the orientation of the robot and recognize the landmarks placed on the mat. The data fusion strategy is described to detect the misclassifications of the landmarks in order to fuse only the reliable information. The orientation given by the OSR algorithm is used to improve significantly the odometry and the recognition of the landmarks is used to reference the odometry to a absolute coordinate system.
\end{abstract}

\section{INTRODUCTION}

ArmAssist is a wireless robot for after stroke upper limb rehabilitation [1]. This project aims at the development of a inexpensive, portable, large-workspace, modular, mobile robotic system for upper-limb functional rehabilitation. The system will implement task-oriented therapy, combining motor recovery and functional recovery. The robot will be attached to the patient forearm, and the patient will perform pre-defined exercises moving it on a table. The device will be used for the treatment of gravity induced disturbance of coordination between shoulder abduction and elbow flexion. Patients using this device would be able to start the rehabilitation program in the care centers and to continue it at home. The system will enable the assessment of patient residual motor/functional ability, implement the physical means for the therapy, and finally assess the results of the therapy. Two important requirements of the robot are: $i$ ) Accurate measurement of the robot in a flat surface and ii) Technologically easy and cheap to implement.

Estimating correctly the position and orientation is one of the most important capabilities for an autonomous mobile platforms. There are many sensors that are being used in mobile robots to determine the robots position and motion: inertial sensors, Differential GPS , sonar, encoders, magnetic compass, radio positioners, laser range finders and also vision based algorithms. Each of them have their own pros and cons. In our device, we wanted to avoid installing any accessory subsystem (beacons, IR landmarks, etc) which leaded to odometry as the only positioning technique.

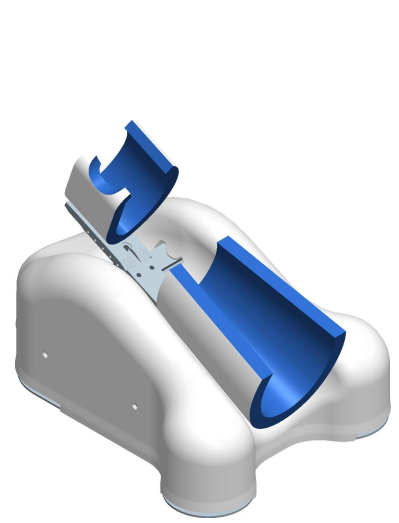

(a) Drawing

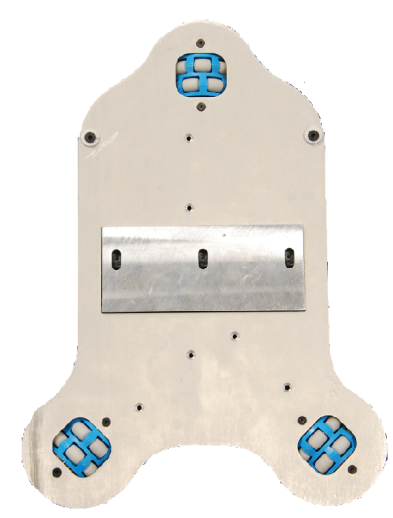

(b) Position of the three mice
Fig. 1: ArmAssist device

Odometry is widely used method to estimate both position and orientation in mobile robots. The core idea is the integration of incremental motion information over time [2][3]. The motion information is usually data from incremental wheel encoders in case of wheeled robots. In our application no actuator is used and installing new sensorized hardware (free wheel with encoder) would make the device more expensive as two encoders and a extra wheel would be needed.

Many techniques have been proposed in order to reduce the effect of the errors [4]. Odometry is considered an accurate short term position estimation method, but as the errors accumulate, the uncertainty of position estimation inevitably increases time.

Another drawback of odometry is that it is unable to provide position information itself and it is normally fused fused with absolute position measurements to reference the odometry with respect to an absolute coordinate system. This independent position information also enables to do odometry corrections, and thus, a more reliable position estimation.

The artificial landmark recognition technique uses the information of location of distinctive landmarks at known locations to obtain position information. This information is fused with the odometry data to correct the odometry errors and to reference the position of the robot with respect to an absolute coordinate system.

The robot's positioning system is equipped with three 
optical mouse sensors. Two mice information of their displacement in two dimensions to the odometry. The third mouse is used as a CCD camera to obtain pictures of the surface in order to extract and recognize visual landmarks of the mat placed below the robot.

The idea of using optical mouse sensors for dead-reckoning for mobile robots is not new [5] [6] [7] [8] [9]. The optical mouse is a very low-cost sensor and has the advantage that the measured displacement is independent from the kinematics of the robot because the optical sensor uses external natural microscopic ground landmarks to obtain the effective relative displacement [10].

\section{METHOD}

This method comprises a optical mouse based odometry, a mouse used as CCD camera with the algorithm to extract and recognize the landmarks on a custom mat and the corrective strategy to detect the misclassifications of the landmarks and fusing of the odometry calculations with the absolute position data.

\section{A. Odometry calculation}

The odometry calculation consists in solving the differential drive kinematics for a two wheel robot. This is a has been solved also non-holonomic wheeled robots with differential steering systems [3]. In our case, the two wheel encoder information have been substituted by two optical mice sensors [10]. The two mice are placed parallel and in a known distance from each other. Mice data are recorded at $125 \mathrm{~Hz}$. At this sampling rate, the mice move very little $(8 \mathrm{~mm}$ at highest speed). It is considered as normal working speed $0.1 \mathrm{~m} / 2$ up to $0.05 \mathrm{~m} / \mathrm{s}$ at slow working speed. This would lead to $0.4 \mathrm{~mm}$ changes per reading. This can lead to very strong changes in trajectory direction. A value was set to $40 \mathrm{~ms}$. Every 5 reading of mice, a new position is calculated using odometry.

\section{B. Absolute position estimation}

We propose absolute position estimation method, to determine in discrete time stamps, the position of the sensor within a custom mat using artificial landmark recognition.

1) Custom printed mat design: The mat spans over the whole range of motion of the arm and contains $\mathrm{N}$ cells which are labeled with a unique label. The cells can be configured in different ways in order to have a finer position estimation in some areas than in others.

In order to make the symbol recognition easy, we created the following symbolic strategy:

- Symbols: Each symbol is a 8x6 matrix and encodes 4 information bits. The information bits 1 to 4 can be black (0) or white (1) and are located in a given position in the symbol as shown in the figure 2a. This way a binary number with values between 0 and 15 is coded in each symbol. The outside black pixels are conceived to generate a uniform grid when repeated throughout the whole mat. This way the detection of the lines will be easier. See details in figure 2.
- Landmarks: Each landmark is composed by two rectangular symbols (see figure 3). There are 16 different symbols so the total possible landmarks would be $16^{2}$ $=256$. But one of the limitations of the optical symbol recognition algorithm (OSR) algorithm is that it can not determine the beginning of the label, thus a restriction is needed in order to have unique information. The highest number will determine the beginning of the label. So, in case the algorithm classifies the landmark as 8,13 this will correspond to the cell 13,8 . With this restriction the total possible combinations is restricted to:

$$
C_{k}^{n}=\frac{n !}{k !(n-k) !}
$$

being $\mathrm{n}=16$ and $\mathrm{k}=2$, there are 60 combinations of symbols, which will allow us to identify 60 different landmarks.

- Cells: Each cell is labeled with a unique landmark that is repeated continuously in that given cell as shown in figure 3. In our case, the landmark is repeated 35 times horizontally and 70 times vertically.

- Mat: The mat is composed by 15 cells per column and 9 cells per row.

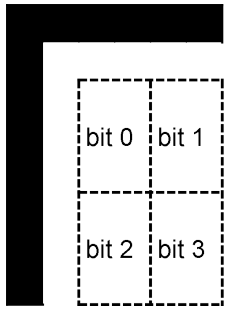

(a) Generic Symbol

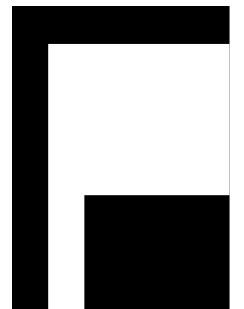

(b) Symbol \#3

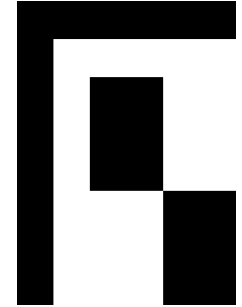

(c) Symbol \#9
Fig. 2: Symbol design

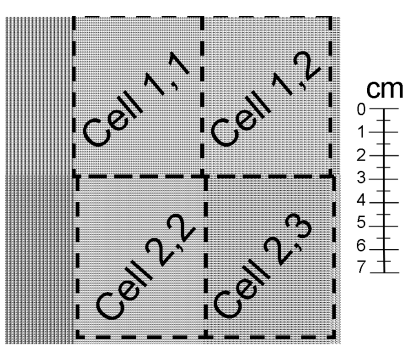

(a) Mat detail

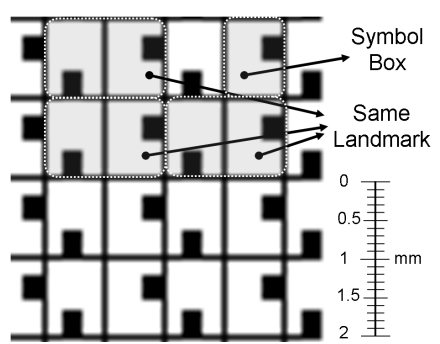

(b) Cell detail
Fig. 3: Mat Design

2) Optical symbol recognition algorithm: The Avago Technologies ADNS-3080 optical mouse sensor has a programmable frame rate over 6400 frames per second. This enables high shutter speeds. In consequence, sharp pictures can be obtained even in high speed conditions. 
The algorithm is divided in the following steps.

- Flat field correction.

- Normalization

- Angle extraction

- Bilinear image rotation and resampling

- Landmark extraction and recognition

- Cell position identification

For explanation purposes we will concentrate in the raw image shown in figure 4a taken with the ADNS-3080 sensor. This image is a very low resolution image ( $30 \times 30$ pixels) and the picture corresponds to an area of $10 \times 12.5 \mathrm{~mm}$.

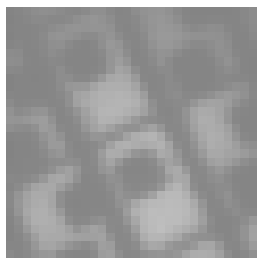

(a) Raw Image

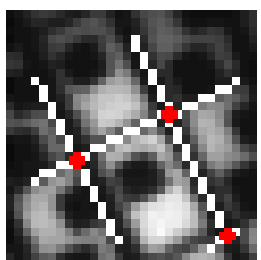

(b) Grid Detection

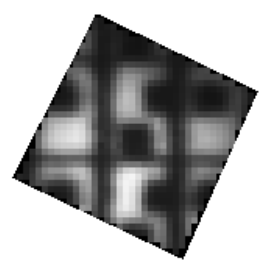

(c) Rotated Image
Fig. 4: Image Rotation Process

- Flat-field correction: Uneven illumination, the LED orientation, dirt/dust on lenses and other factors can result in a poor quality image. The flat field image should, ideally, be a field of view of the mat without any symbols in it.

- Normalization: The flat-field corrected image is then normalized to numbers between 10 (nearly black) and 240 (nearly white). This is done so in order NOT to have pixels with value zero, as the zero value is reserved for not computed for the rotation process.

- Angle extraction: The way the boxes were designed draw a grid of continuous black lines in the captured frame. The algorithm to detect this lines is a basic form of the Hough transform [11][12]. Instead of searching and voting for a single line this algorithm searches for multiple lines. The main lines are parallel to each other in a known distance from each other (the width of a symbol picture), and the secondary lines are placed perpendicular to the main lines, and the distance between secondary lines is equal to the height of the symbol picture. This algorithm searches for the location and orientation of the grid. Note that each of the line crossing points is a correct translation of the grid (marked in red in figure 4), which means that the solution of the problem is not unique. This voting procedure is carried out in a parameter space, from which candidates are obtained. In our case the parameter space is (horizontal translation of grid crossing point -c-, vertical translation of grid crossing point -r- and angle of the main grid lines $\theta$ ). On the other hand we can ensure that there is a zone smaller than the whole picture, where there is a solution. This way, the algorithm does not have to scan through all the picture pixes searching for the solution, and it is limited to search in a smaller central area $(15 \times 15$ pixels $)$. If the 10 most voted candidate's result are withing a maximum error of 10 degrees, the most voted solution is accepted and the pictures is used for landmark detection. Otherwise, the image is rejected.

- Bilinear image rotation and resampling: The final goal is to rotate around the center of the image for landmark extraction and recognition. As the footprint of the image remains constant, the size (in pixels) of the landmark is known. The central area of the size of the landmark is extracted.

- Landmark extraction and recognition: In order to separate each symbol from each other, it is necessary first to resample the image. The image is resampled to the double of the resolution of a theoretical landmark (24 columns and 16 rows). Then, it is necessary to re-shift the image into the correct position. This is done using the information box design. All landmarks start with a black column, and 6 columns after (12 columns after in the resampled image), another black column should be present. This is used to determine which column is the first one. Similarly the row re-shifting is done. Once the landmark picture is re-shifted is easy to locate the areas where the symbol bits are and classify them as black or white.

- Cell position identification: The cell position in the mat is known using a lookup table.

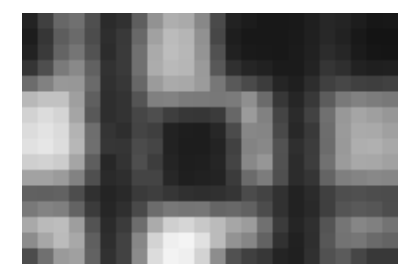

(a) Raw Image

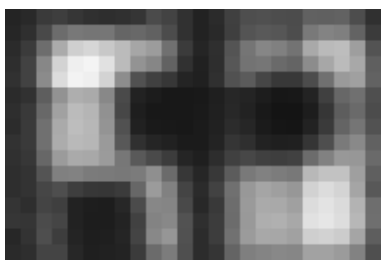

(b) Grid Detection
Fig. 5: Landmark extraction and recognition

\section{Odometry and absolute position correction fusing strategy}

The optical mouse sensors are very sensitive to light conditions and distance from lens reference plane to navigation surface. The light conditions remain constant during working time and this is automatically adjusted by the sensors chip. Mice work better in less ideal surfaces (e.g. porous surfaces, uneven surfaces) as the chip is able to detect more features of the surface. This is given by the SQUAL (surface quality) parameter. Its average value on white paper is 75 (in optimal hight adjustment) and the maximum value is 169 [13]. Due to the mat design, the number of features with which the mouse chip estimates its motion is very high (SQUAL = $131.32 \pm 6.84)$.

The systematic errors in the odometry of our system are mainly different height placement of the sensors and misalignment of the sensors, and there are many strategies to correct systematic odometry errors [14].

Another error correction source is the redundancy of the mice data. As the mice are solidly attacked to each other, the 
readings of the mice along the axis that joins the centers of the two sensors should be equal. This redundancy can be used for error detection and reduction for better odometry estimation [7].

On the first version of the 3 optical mouse sensor based positioning system, non of the previously mentioned systematic odometry error correction strategy has been implemented. The angle estimation errors are the core source of position estimation error calculated by odometry. The odometry correction we present focuses on orientation error correction. The method focuses on redundant source of orientation changes. As stated before, odometry is a reliable accurate short term position estimation method and therefore the orientation changes in a short period of time are very reliable.

The OSR algorithm does not always return a value (section II-B2), thus, the absolute orientation information $\theta_{A}$ has an asynchronous sampling rate. First, the relative orientation values sampled at $25 \mathrm{~Hz}\left(\theta_{R}(n)\right)$ is subsampled to get the information in the OSR data time slots $\left(\theta_{R}(\hat{n})\right)$. Once this is done, the consistency between the absolute orientation $\theta_{A}(\hat{n})$ and the relative orientation $\theta_{R}(\hat{n})$ is checked: if the information of the relative orientation variation (equation 1) matches within boundaries $(T h \theta)$ with the changes of the absolute orientation variation (2) for the last four valid images (3), the absolute orientation information is defined as compliant, and used to determine the actual orientation error as shown in (4).

$$
\begin{array}{r}
\Delta \theta_{R}(\hat{n})=\theta_{R}(\hat{n})-\theta_{R}(\hat{n}-1) \\
\Delta \theta_{A}(\hat{n})=\theta_{A}(\hat{n})-\theta_{A}(\hat{n}-1) \\
{\left[\left|\Delta \theta_{R}(\hat{n}-i)-\Delta \theta_{A}(\hat{n}-i)\right|\right]_{i=0,1,2,3}<T h \theta} \\
\epsilon(\hat{n})=\frac{\sum_{i=1}^{4} \theta_{R}(\hat{n}-i)-\sum_{i=1}^{4} \theta_{A}(\hat{n}-i)}{4}
\end{array}
$$

Although the way of consistency rules of absolute position data change, the same method can be used for position data consistency checking and correction if extended to two dimensions. First, the relative position values $P_{R}(n)$ are subsampled to get the information in the OSR data time slots $P_{R}(\hat{n})$. The traveled distance between two time slots $\hat{m}$ and $\hat{n}$ will be given by (6) and (7).

$$
\begin{gathered}
P_{R}(n)=\left[\begin{array}{l}
X_{R}(n) \\
Y_{R}(n)
\end{array}\right], P_{A}(\hat{n})=\left[\begin{array}{l}
X_{A}(\hat{n}) \\
Y_{A}(\hat{n})
\end{array}\right] \\
\Delta X_{R}^{\hat{m}, \hat{n}}=X_{R}(\hat{m})-X_{R}(\hat{n}) \\
\Delta Y_{R}^{\hat{m}, \hat{n}}=Y_{R}(\hat{m})-Y_{R}(\hat{n}) \\
\Delta P_{R}^{\hat{m}, \hat{n}}=\sqrt{\left(\Delta X_{R}^{\hat{m}, \hat{n}}\right)^{2}+\left(\Delta Y_{R}^{\hat{m}, \hat{n}}\right)^{2}} \\
\Delta X_{A}^{\hat{m}, \hat{n}}=X_{A}(\hat{m})-X_{A}(\hat{n}) \\
\Delta Y_{A}^{\hat{m}, \hat{n}}=Y_{A}(\hat{m})-Y_{A}(\hat{n}) \\
\Delta P_{A}^{\hat{m}, \hat{n}}=\sqrt{\left(\Delta X_{A}^{\hat{m}, \hat{n}}\right)^{2}+\left(\Delta Y_{A}^{\hat{m}, \hat{n}}\right)^{2}}
\end{gathered}
$$

The matrices $\mathbf{R}(8)$ and $\mathbf{A}(9)$ are $\mathrm{k} \times \mathrm{k}$ matrices that contain the distances between all points $P_{R}(\hat{n})$ and $P_{A}(\hat{n})$ respectively being $\mathrm{k}$ the number of successfully processed pictures. The matrix $\mathbf{E}$ is the matrix of the mismatch between relative and absolute positions.

$$
\begin{array}{r}
\mathbf{R}_{i, j}=\Delta P_{R}^{i, j} \\
\mathbf{A}_{i, j}=\Delta P_{A}^{i, j} \\
\mathbf{E}_{i, j}=\mathbf{R}_{i, j}-\mathbf{A}_{i, j}
\end{array}
$$

Finally the consistency between absolute positions and the relative positions is checked. This is done using the validation vector $\mathbf{V}$ that contains the number of consistent points assuming that $P_{A}(i)$ is correct (12).

$$
\begin{gathered}
\mathbf{C}_{i, j}=\left\{\begin{array}{c}
1 \text { if } E_{i, j}<T h \Delta \forall i, j \in(0, k) \\
0 \text { if } E_{i, j} \geq T h \Delta \forall i, j \in(0, k)
\end{array}\right. \\
V_{j}=\sum_{i=0}^{k} C_{i, j}
\end{gathered}
$$

The index $\hat{s}$ of the maximum value of the vector $V_{j}$ corresponds to the the absolute position $P_{A}(\hat{s})$ that gave most of the consistent absolute position information. The set of absolute positions $P_{A}(\hat{S})$ where $C_{\hat{s}, \hat{S}}$ has the value 1 is the set that will be used for position error calculation $\epsilon_{X}$ and $\epsilon_{Y}$ as shown in (13). This is done only if the size of the matrices $\mathbf{R}$ and $\mathbf{A}$ is higher than 16, thus when the orientation estimation of at least 16 images are consistant with the estimation of the orientation given by odometry.

$$
\begin{aligned}
\epsilon_{X} & =\overline{X_{R}(\hat{S})}-\overline{X_{A}(\hat{S})} \\
\epsilon_{Y} & =\overline{Y_{R}(\hat{S})}-\overline{Y_{A}(\hat{S})}
\end{aligned}
$$

\section{RESULTS}

\section{A. Optical mouse reliability}

In the characterization of the optical mouse behavior, straight trajectories were carried out manually in slow, medium and fast movements. As the robot aims after stroke upper limb rehabilitation the maximum working speed is estimated at $1 \mathrm{~m} / \mathrm{s}$. The tests were carried out in $0.1 \mathrm{~m} / \mathrm{s}, 0.5 \mathrm{~m} / \mathrm{s}$ and $1 \mathrm{~m} / \mathrm{s}$ speeds.

TABLE I: Spacial Resolution at Different Speeds

\begin{tabular}{c|cc} 
Speed $[\mathrm{m} / \mathrm{s}]$ & CPI mouse \# 1 & CPI mouse \# 2 \\
\hline 0.1 & $1435.6 \pm 14.8$ & $1251.2 \pm 21.8$ \\
0.5 & $1452.1 \pm 31.3$ & $1185.6 \pm 47.4$ \\
1 & $1501.7 \pm 22.3$ & $1229.6 \pm 28.2$
\end{tabular}




\section{B. Orientation correction}

The orientation detection algorithm with OSR has been tested. The images of a database of 100 images corresponding to a random trajectory of 30 seconds were used to determine the accuracy of the OSR orientation data. For each of the images an optimal solution was given and compared with the output of the OSR algorintm. The error in absolute value of the results of the OSR algorithm after the consistency check was $2.81 \pm 1.57$ degrees of an angle (maximum was 5.18 and minimum was 0.04 [deg]). The number of frames with processed angle data was 90 and 70 frames were consistent in relative and absolute orientation changes.

As the most important corrective action concerns orientation correction, a test has been carried out to quantify the odometry errors due to orientation drift. The robot was manually moved following a square shaped trajectory finishing at the starting point. The following parameters have mean measured: Test Duration (TD), length of the trajectory with orientation correction $(\mathrm{OC})$ and the trajectory without orientation correction (NOC), mean root square difference between the positions of the trajectory with and without orientation corrections (MRSD), the total length of the trajectories (Length), the final points distance to the initial point (Distance to SP), and the final point's estimation improvement due to orientation correction factor (Improvement) (table II). All values are given in millimeters.

TABLE II: Orientation Correction Effect Test Results

\begin{tabular}{ccccccc}
\multirow{2}{*}{ Test \# } & \multicolumn{2}{c}{ Length } & \multirow{2}{*}{ MRSD } & \multicolumn{2}{c}{ Distance to SP } & \multirow{2}{*}{ Improvement } \\
\cline { 2 - 3 } & OC & NOC & & OC & NOC & \\
\hline 1 & 1226 & 1226 & 12.42 & 81.14 & 168.80 & 87.66 \\
2 & 1225 & 1225 & 36.16 & 89.44 & 191.57 & 102.13 \\
3 & 1237 & 1237 & 40.31 & 40.07 & 178.64 & 138.56 \\
4 & 1048 & 1232 & 24.70 & 180.38 & 291.90 & 111.52 \\
5 & 1121 & 1364 & 28.84 & 145.80 & 267.01 & 121.20 \\
6 & 1141 & 1212 & 33.16 & 114.50 & 217.65 & 103.16 \\
7 & 1238 & 1238 & 30.02 & 45.44 & 161.03 & 115.59 \\
8 & 1213 & 1213 & 32.03 & 52.50 & 160.07 & 107.57 \\
9 & 1213 & 1213 & 38.86 & 39.38 & 167.91 & 128.53 \\
10 & 1216 & 1288 & 42.16 & 44.51 & 183.28 & 138.76 \\
\hline Mean & 1187.8 & 1244.8 & 31.9 & 83.3 & 198.8 & -115.5 \\
SD & 63.3 & 47.4 & 8.7 & 49.5 & 46.1 & 16.5
\end{tabular}
6.

As an example, the output of the test \# 7 is shown in figure

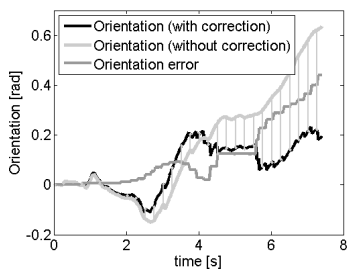

(a) Angle drift

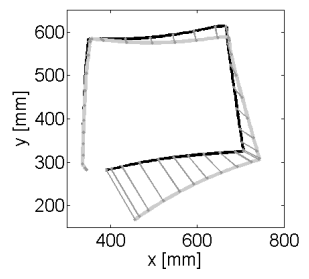

(b) Position difference
Fig. 6: Position and orientation differences due to orientation correction during test \#7

\section{Position correction}

The position estimation of the OSR algorithm was also tested using the database of 100 images. These images of different landmarks in different solutions were tagged manually and compared to the output of the ORS algorithm As it was said before only 70 pictures of these images were further treated for feature extraction and landmark recognition. The number of correct landmark recognition is $18 \%$ which is significantly better than a random classifier which would correctly classify one out of 60 different landmarks (3\%). After applying the consistency check rule only 7 pictures were processed. The accuracy using this 7 pictures is up to 85.71 $\%(6 / 7)$ and the remaining images was misclassified with the neighbour landmark.

Another test with 10 trials was done to verify the accuracy of the absolute position correction. When the 16 images are consistent with the estimation of the orientation given by odometry the the odometry is referenced in an absolute reference system for the first time. At this moment the position of the armassist was measured and compared to the corrected position.

\section{CONCLUSION}

The study showed similar behavior in slow, medium and fast movements in spacial resolution but quite different from one sensor to another. However, the analysis concluded that even a very cheap optical mouse sensors can be sufficiently accurate, precise and reliable to perform a optical mouse sensor based short term odometry for the ArmAssist robot.

Concerning OSR performance in orientation detection, the modified Hough transform correction strategy, and consistency check showed very good results. The OSR orientation is very reliable as $100 \%$ of the results after the concistency check rule have an error below 5 degrees. Additionally, if this data is used to correct the odometry, the position error (in $\frac{\mathrm{mm}}{\mathrm{m}}$ ) decreases significanly by $58.10 \%$.

Regarding the OSR performance in landmark recognition the accuracy of $18 \%$ could seem rather low. The raw images have very poor quality and contrast (see figure 4 ). On the other hand the consistency check rule improves the accuracy to 90 $\%$, but the number of pictures that fullfill the consistency check rule are very few, so the time between two absolute position corrections could be rather large (7 correction calculations in 30 seconds).

We can conclude that even with very few corrective strategies have been implemented until now, the proposed navigation system can be used in desktop mobile robots where the range of motion of the robot is limited. This method enables the construction of a cheap global positioning system where the main drawback of unbounded error accumulation of odometry is solved.

\section{ACKNOWLEDGMENT}

This work was supported in part by the FIK Project, San Sebastian, Spain. 


\section{REFERENCES}

[1] J. Perry, H. Zabaleta, A. Belloso, and T. Keller, "ARMassist: A lowcost device for telerehabiltation of post-stroke arm deficits," in World Congress on Medical Physics and Biomedical Engineering, September 7-12, 2009, Munich, Germany. Springer, 2009, pp. 64-67.

[2] J. Borenstein, H. Everett, and L. Feng, "Where am I? Sensors and methods for mobile robot positioning," University of Michigan, vol. 119, p. $120,1996$.

[3] J. Crowley, "Control of translation and rotation in a robot vehicle," in Proceedings of the IEEE Conference on Robotics and Automation, 1989.

[4] Y. Tonouchi, T. Tsubouchi, and S. Arimoto, "Fusion of dead-reckoned positions with a workspace model for a mobile robot by Bayesian inference," in Intelligent Robots and Systems' 94.'Advanced Robotic Systems and the Real World', IROS'94. Proceedings of the IEEE/RSJ/GI International Conference on, vol. 2. IEEE, 2002, pp. 1347-1354.

[5] D. Sekimori and F. Miyazaki, "Precise dead-reckoning for mobile robots using multiple optical mouse sensors," Informatics in Control, Automation and Robotics II, pp. 145-151, 2007.

[6] A. Roskilly and N. Tunwattana, "Investigations into the effects of illumination and acceleration on optical mouse sensors as contact-free 2D measurement devices," 2009.

[7] A. Bonarini, M. Matteucci, and M. Restelli, "Automatic error detection and reduction for an odometric sensor based on two optical mice," in Robotics and Automation, 2005. ICRA 2005. Proceedings of the 2005 IEEE International Conference on. IEEE, 2006, pp. 1675-1680.

[8] J. Palacin, I. Valganon, and R. Pernia, "The optical mouse for indoor mobile robot odometry measurement," Sensors and Actuators A: Physical, vol. 126, no. 1, pp. 141-147, 2006.

[9] S. Lee, "Mobile robot localization using optical mice," in Robotics, Automation and Mechatronics, 2004 IEEE Conference on, vol. 2. IEEE, 2005, pp. 1192-1197.

[10] A. Bonarini, M. Matteucci, and M. Restelli, "A kinematic-independent dead-reckoning sensor for indoor mobile robotics," in Intelligent Robots and Systems, 2004.(IROS 2004). Proceedings. 2004 IEEE/RSJ International Conference on, vol. 4. IEEE, 2005, pp. 3750-3755.

[11] R. Duda and P. Hart, "Use of the Hough transformation to detect lines and curves in pictures," Communications of the ACM, vol. 15, no. 1, pp. $11-15,1972$.

[12] "Method and means for recognizing complex patterns," 1962, uS Patent $3,069,654$.

[13] A. Datasheet, "ADNS-3080 Data Sheet Avago Technologies," 2008.

[14] J. Borenstein and L. Feng, "Measurement and correction of systematic odometry errors in mobile robots," Robotics and Automation, IEEE Transactions on, vol. 12, no. 6, pp. 869-880, 2002. 